\title{
Raloxifene Hydrochloride
}

National Cancer Institute

\section{Source}

National Cancer Institute. Raloxifene Hydrochloride. NCI Thesaurus. Code C1762.

The hydrochloride salt form of raloxifene, a selective benzothiophene estrogen receptor modulator (SERM) with lipid lowering effects and activity ag ainst osteoporosis. Raloxifene hydrochloride specifically binds to estrogen receptors in responsive tissue, including liver, bone, breast, and endometrium. The resulting lig and-receptor complex is translocated to the nucleus where, depending on the tissue type, it promotes or suppresses the transcription of estrogen-regulated genes, thereby exerting its agonistic or antagonistic effects. This agent functions as an estrogen agonist in lipid metabolism, thereby decreasing total and LDL cholesterol levels. In tissue like bone, it decreases bone resorption and bone turnover and increases bone mineral density. Raloxifene hydrochloride acts as an estrogen antagonist in uterine and breast tissue. This agent also exerts an anti-proliferative effect on estrogen-sensitive breast cancer. 